\title{
PROGRAMA DE EDUCAÇÃO PARA O TRABALHO E VIGILÂNCIA EM SAÚDE
}

\author{
EDUCATION FOR WORK AND HEALTH SURVEILLANCE PROGRAM
}

\author{
Maria Socorro Carneiro Linhares ${ }^{1}$ \\ Cibelly Aliny Siqueira Lima Freitas ${ }^{2}$ \\ Ana Karine Macedo Teixeira ${ }^{3}$ \\ Raimundo Vieira Dias ${ }^{4}$ \\ Sandra Maria Carneiro Flor ${ }^{5}$ \\ João Sérgio Araújo Soares ${ }^{6}$ \\ Francisco Roger Aguiar Cavalcante ${ }^{7}$
}

Resumo As iniciativas nacionais que se propõem contribuir para a reorientação da formação e do modelo de atenção nos levaram a descrever a experiência de implantação do Programa de Educação para o Trabalho (PET)-Saúde/Vigilância em Saúde (VS) em Sobral, Ceará. Trata-se de estudo descritivo, do tipo relato de experiência, vivenciado por estudantes dos cursos de enfermagem da Universidade Estadual Vale do Acaraú (UVA) e medicina e odontologia da Universidade Federal do Ceará (UFC), tutores e preceptores do PET-Saúde/VS. As informações foram registradas pelos tutores e preceptores durante as atividades desenvolvidas desde a implantação do programa (julho de 2010). O processo de trabalho desempenhado no PET-Saúde/VS compreende três eixos estruturantes que se intercomunicam, constando vivências teórico-conceituais, vivências no serviço e vivências de pesquisa. Para garantir a interface ensino-serviço e a integração entre as ações de vigilância em saúde e atenção básica, construímos uma 'tecnologia' pedagógica que chamamos de tecnologia de educação permanente na vigilância em saúde. A experiência permitiu aos estudantes uma visão mais ampla, acerca das condições de saúde da população, da importância do conhecimento sobre problemas locais para o trabalho da vigilância em saúde, a fim de prestar assistência qualificada e voltada para a realidade de cada território.

Palavras-chave vigilância em saúde; equipe interdisciplinar; educação profissional em saúde; atenção primária à saúde.

\begin{abstract}
The Brazilian initiatives that propose to contribute to redirecting training and the care model led us to describe the experience of deploying of the Education for Work Program (PET) - Health/Health Surveillance (HS) in Sobral, northeastern Brazil. This is a descriptive, experience-reporting study, experienced by nursing students at the Vale do Acaraú State University (UVA) and medicine and dentistry students at the Federal University of Ceará (UFC), tutors, and PET-Health/HS preceptors. The information was recorded by the tutors and preceptors during the activities undertaken ever since the program was deployed (July 2010). The work process performed under PET-Health/HS comprises three intercommunicating structural axes, consisting of theoretical and conceptual experiences, experiences in service, and research experiences. To ensure the interface between teaching and service and integration between health surveillance and primary care, we built an educational 'technology' we call a permanent education in health surveillance technology. The experience afforded the students a broader view about the population's health conditions and on the importance of understanding local issues for health surveillance in order to provide qualified care targeted at the reality at each territory. Keywords health surveillance; interdisciplinary team; health professional education; primary health care.
\end{abstract}




\section{Introdução}

No Brasil, o Sistema Único de Saúde (SUS) foi proposto e implementado trazendo grandes avanços na gestão e na organização dos serviços de atenção à saúde. No contexto epidemiológico e demográfico, apesar dos grandes desafios, observa-se uma importante melhoria de indicadores, como progressiva redução da mortalidade infantil e modificação da estrutura etária da população. Ao mesmo tempo, a saúde passou a ser um direito do cidadão e um dever do Estado, e a sociedade passou a ter vários canais de participação na gestão dos sistemas e serviços de saúde, além de maior acesso às informações (Brasil, 2001).

Nesse contexto se insere a vigilância à saúde ou vigilância em saúde, que pode ser definida como a observação contínua da distribuição e das tendências da incidência de doenças mediante coleta sistemática, consolidação e avaliação de informes de morbidade e mortalidade (Waldman, 1998).

Entretanto, percebe-se o quanto é desafiador o diálogo dos profissionais que integram as equipes de saúde da família e da vigilância em saúde como instrumento de detecção, avaliação e planejamento que possibilitam tomadas de decisões para minimizar, eliminar ou prevenir agravos na saúde coletiva, bem como desenvolver ações de promoção da saúde.

O desafio é ainda maior quando se trata da integração ensino-serviço. Compreende-se que a aproximação ensino-serviço é imprescindível para que se materializem transformações efetivas na formação de recursos humanos no campo da saúde no Brasil, bem como para que a produção de conhecimento cause impacto significativo nos indicadores de saúde e na qualidade da atenção prestada no SUS.

O Ministério da Saúde, por meio da Secretaria de Gestão do Trabalho e da Educação na Saúde (SGTES), vem desenvolvendo ações relativas a um amplo processo de formação e qualificação dos profissionais de saúde e de regulação profissional no âmbito do SUS, visando à execução de uma política nacional de educação permanente em saúde (Costa e Miranda, 2009).

Nesse sentido, o Projeto UNI (Nova Iniciativa na Formação dos Profissionais de Saúde), o VerSUS (Vivência Estágio na Realidade do Sistema Único de Saúde), o Pró-Saúde (Programa Nacional de Reorientação da Formação Profissional em Saúde), o AprenderSUS (o SUS e os cursos de graduação na área da saúde), bem como propostas do Programa de Educação pelo Trabalho para a Saúde (PET-Saúde) e do PET-Saúde/Vigilância em Saúde, são exemplos expressivos da tentativa de aproximação entre ensino, serviço e comunidade, mediante a consecução de avanços nos projetos pedagógicos articulados com práticas de saúde e princípios do SUS. A integração ensino-serviço surge então como mediadora de diálogos, reflexões e aprendizados múltiplos, em que a vigilância em saúde caracteriza-se como um dos cenários desse aprendizado transformador (Seta e Reis, 2009). 
Assim é que iniciativas de âmbito nacional que se propõem a contribuir para a reorientação da formação e do modelo de atenção, a exemplo do Pró-Saúde, do PET-Saúde e do PET-Saúde/Vigilância em Saúde, têm se conformado com propostas singulares, as quais se configuram como caminhos que se descortinam para essa transformação e produção da autonomia dos diversos atores envolvidos na integração ensino-serviço-comunidade.

Este artigo se propõe descrever a experiência de implantação do PET-Saúde/Vigilância em Saúde de Sobral, no Ceará. Para tanto, realizou-se um estudo descritivo (Minayo, 2000), do tipo relato de experiência, vivenciado por estudantes dos cursos de enfermagem da Universidade Estadual Vale do Acaraú (UVA) e medicina e odontologia da Universidade Federal do Ceará (UFC), bem como tutores e preceptores participantes do PET-Saúde/Vigilância em Saúde de Sobral, desde a implantação do projeto, em julho de 2010.

O cenário da experiência é o território de saúde de Sobral, no Ceará, envolvendo os centros de saúde da família e os serviços de vigilância em saúde. Sobral integra uma rede assistencial hierarquizada e regionalizada do SUS naquele estado. Sua posição como município-sede para a Microrregional de Sobral e Polo da Macrorregião Norte do Ceará atende aos princípios da regionalização da saúde no estado.

A base para a organização do Sistema de Saúde de Sobral norteia-se nos princípios e nas diretrizes do SUS. Esse modelo tem como um dos seus pilares a Estratégia Saúde da Família (ESF). No ano de 2012 existiam 48 equipes da ESF, sendo 34 na sede e 14 nos distritos. Essas equipes atuam em 27 centros de saúde da família (14 na sede e 13 nos distritos), distribuídos em áreas compreendidas como territórios, onde, dependendo de sua localização e do perfil social e epidemiológico da população, acompanham de 600 a 1.200 famílias.

As informações deste estudo foram registradas pelos tutores e preceptores do PET-Saúde/VS durante as atividades desenvolvidas com os estudantes desde a implantação do programa, em julho de 2010, e estão apresentadas de forma descritiva, contemplando implementação, proposta metodológica e processo de acompanhamento e avaliação.

Esta pesquisa cumpriu a resolução do Conselho Nacional de Saúde n. 196, de outubro de 1996, que dispõe como princípios básicos da bioética: autonomia, não maleficência, justiça e equidade (Brasil, 1996).

\section{A experiência do PET-Saúde/VS em Sobral}

\section{0 que é o projeto?}

O Programa de Educação pelo Trabalho para a Saúde na área da Vigilância em Saúde é um programa instituído no âmbito dos ministérios da Saúde 
e da Educação por intermédio das portarias interministeriais n. 421/MS/MEC, de 3 de março de 2010, e portaria conjunta n. 3/SG-TES/SVS/SESu, de 3 de março de 2010, que se destina a viabilizar o aperfeiçoamento e a especialização em serviço, bem como a iniciação ao trabalho, estágios, pesquisas e vivências, dirigidos, respectivamente, aos profissionais e estudantes da área da saúde, considerando as necessidades do SUS na área de vigilância em saúde.

Visando à seleção de projetos para o PET-Saúde/VS, as instituições de ensino superior (IES) Universidade Federal do Ceará (UFC) e Universidade Estadual do Vale do Acaraú (UVA), em parceria com a Secretaria Municipal de Saúde de Sobral, se apresentaram como proponentes para participarem do programa.

Considerando a necessidade de realizar a integração entre cursos de graduação da área de saúde, numa perspectiva de ensino-serviço, tendo o serviço público como cenário de prática multidisciplinar e interdisciplinar e de interação de forma cooperativa e ética, a UVA, a UFC e a Secretaria de Saúde de Sobral elaboraram um único projeto para o PET-Saúde/VS, envolvendo os cursos de medicina, odontologia, enfermagem e educação física. O projeto apresentado para seleção propôs seis grupos, tendo sido aprovados apenas dois. Com o objetivo de atender às adequações do PET-Saúde/VS, foram definidos os critérios de vagas para os cursos das IES e abrangência das áreas da vigilância em saúde local.

O contexto epidemiológico de Sobral configurou-se como importante ferramenta para a orientação das vagas de estudantes por cursos em cada IES e da abrangência do PET-Saúde/VS nos serviços da vigilância em saúde do município.

Como critérios para garantir a distribuição equânime das vagas por cursos das instituições de ensino superior proponentes, foi dada maior relevância à participação de conteúdos pedagógicos na área de vigilância em saúde. Nesse caso, o curso de enfermagem ficou com oito vagas, e os cursos de medicina e de odontologia da UFC, com quatro vagas cada.

Para selecionar preceptores dos serviços da Vigilância em Saúde de Sobral, foram consideradas as áreas com maiores problemas de saúde, atentando aos indicadores do Pacto pela Saúde, da Programação das Ações de Vigilância em Saúde (PAVS) e com maior possibilidade de integração com a atenção básica.

As áreas escolhidas foram a vigilância epidemiológica, que compreende o conjunto de ações de vigilância e controle das doenças transmissíveis, não transmissíveis e agravos; e a vigilância dos fatores biológicos de risco, que compreende o conjunto de ações que proporcionam a identificação e a potencialidade de risco dos vetores, pragas urbanas, fauna sinantrópica e animais peçonhentos, com finalidade de prevenir e controlar zoonoses, doenças endêmicas, doenças transmitidas por vetores por meio de intervenções dos 
condicionantes individuais, coletivos e ambientais responsáveis pela atuação sobre os determinantes sociais da saúde. Ressalta-se que a escolha foi realizada juntamente com a Coordenação da Vigilância em Saúde de Sobral, a qual realiza análise permanente da situação de saúde e acompanhamento das tendências do padrão sanitário da população, atuando de forma integrada com a atenção básica.

Nesse processo de implementação da proposta PET-Saúde/VS em Sobral, destaca-se uma estratégia que vem orientando os processos de trabalho do sistema local de saúde, a qual é o reconhecimento desse sistema como Sistema Saúde Escola de Sobral. Trata-se de uma estratégia e, ao mesmo tempo, de um modo de conceber os processos de trabalho que ocorrem no interior da rede de saúde local segundo uma lógica em que o conjunto das experiências e trocas humanas que se dão no território é reconhecido como possuindo valor pedagógico, ou seja: aprender é algo que se dá regularmente no cotidiano dos profissionais da Secretaria da Saúde e Ação Social de Sobral. Esse aprender, por sua vez, é potencializado na medida em que se tem como pressuposto básico a proposta da Educação Permanente e da Educação Popular e, ainda, a sensível articulação entre serviço, ensino e pesquisa. Esse sistema aprendente, tanto quanto um modelo, compreende também um método que resulta na constituição de uma comunidade aprendente (Pinto et al., 2008).

No sistema de saúde de Sobral, a aposta fundamental da Educação Permanente é de que os processos educativos devem ter como fim a organização dos serviços mediante sua análise e a solução dos problemas identificados.

A perspectiva oferecida pela Educação Popular, inspirada principalmente na abordagem freireana da educação, baseia-se na dialogicidade, na horizontalidade das relações e no profundo respeito pelo saber do educando (Dias et al., 2007).

Nesse cenário, a Escola de Formação em Saúde da Família Visconde de Saboia (EFSFVS), escola técnica do SUS, sediada em Sobral, coordena processos de educação permanente, entendendo que estes contribuirão para ganhos de maiores níveis de resolutividade, qualidade e humanização dos serviços.

\section{Proposta metodológica}

O processo de trabalho desempenhado no PET-Saúde/VS de Sobral visa à integração entre as universidades e o serviço com uma carga horária de oito horas semanais, compreendendo três eixos estruturantes que se intercomunicam, constando: vivências teórico-conceituais, vivências no serviço e vivências de pesquisa.

As vivências teórico-conceituais dizem respeito à formação e à qualificação teórica entre estudantes de graduação, preceptores e tutores no que tange aos conceitos, práticas e ações da vigilância em saúde. Considera-se 
também o conteúdo didático dos módulos/disciplinas dos cursos de medicina, enfermagem e odontologia, o qual se articula com as vigilâncias (Epidemiológica, Sanitária, Ambiental, Nutricional, dos Fatores Biológicos de Risco, Saúde do Trabalhador), análise de situação em saúde e promoção da saúde. As atividades teórico-conceituais ocorrem de forma processual orientada com base nas demandas do serviço e nas necessidades dos integrantes do PET-Saúde/VS, já que estes trazem consigo um conhecimento prévio sobre vigilância em saúde que será ressignificado com as vivências proporcionadas pelo projeto.

As vivências no serviço compreendem todas as ações de vigilância em saúde realizadas pelos acadêmicos em conjunto com os preceptores nos serviços de saúde e no seu território de abrangência. Essas práticas foram distribuídas de acordo com a estruturação física e com cenários das práticas da vigilância em saúde do Sistema Municipal de Saúde de Sobral, abrangendo prioritariamente a vigilância epidemiológica e a vigilância dos fatores biológicos de risco.

Dentre as atividades propostas, estão: atividades de campo (investigação e inquéritos epidemiológicos); análise epidemiológica situacional dos principais agravos e riscos à saúde da população no que se refere às doenças transmissíveis e não transmissíveis, além dos fatores biológicos de risco; desenvolvimento de ações de vigilância em saúde que possibilitem minimizar, eliminar ou prevenir riscos à saúde e capacitação dos profissionais da atenção básica para as ações de vigilância em saúde no território. As atividades de capacitação envolviam temáticas sobre a vigilância de doenças como tracoma, hanseníase, tuberculose, dengue e leishmaniose, entre outras.

Outra proposta de vivência nos serviços foi a socialização com a população das informações sobre saúde e dos principais agravos existentes em seu território e nos espaços dos Centros de Saúde da Família, por meio de reuniões em grupos e da elaboração de um boletim epidemiológico - produto do trabalho do PET/VS - contendo informações da epidemiologia local.

Já as vivências de pesquisa compreendem a realização de trabalhos científicos apresentados em eventos nacionais, como o Congresso Brasileiro de Epidemiologia, promovido pela Associação Brasileira de Saúde Coletiva (Abrasco), o Congresso Brasileiro de Doenças Sexualmente Transmissíveis (DST)/Aids e eventos regionais e locais, conforme as normas acadêmicas e os preceitos éticos. Os trabalhos produzidos também foram publicados em periódicos com classificação no Qualis Capes. As vivências proporcionaram uma reflexão sobre as práticas em serviço dos integrantes do PET-Saúde/VS, possibilitando uma avaliação de tecnologias desenvolvidas, além da divulgação no meio científico das ações de vigilância em saúde realizadas.

Nesse processo de ensino-aprendizagem, tutores, preceptores e estudantes desempenharam papéis importantes para o êxito do Programa PET/VS 
em Sobral. As vivências do PET/VS têm sido desenvolvidas considerando uma tecnologia leve de educação descrita a seguir.

\section{Tecnologia de educação em serviço na vigilância em saúde}

O desafio de operacionalizar o projeto PET-Saúde/VS propiciou o surgimento de uma nova 'tecnologia' pedagógica representada na Figura 1, que se denominou de tecnologia de educação em serviço na vigilância em saúde, apoiando-se no conceito de tecnologia leve de Merhy (2002).

Trata-se de cinco momentos pedagógicos que obedecem a um fluxo e que se retroalimentam, além de se intercomunicarem com as ações de vigilância em saúde e com as ações da atenção básica, tendo a pesquisa científica presente de modo transversal durante todo o processo.

Os estudantes iniciam a atividade fazendo a análise situacional por meio do estudo dos bancos de dados dos sistemas de informação, de relatórios da vigilância epidemiológica do município e de investigação direta de campo, com o objetivo de conhecer e analisar o comportamento epidemiológico das condições de saúde do município, buscando sempre fazer comparações nos âmbitos regional e nacional.

O segundo momento pedagógico consta da busca de referencial teórico - manuais, documentos ou normas técnicas - que permita um embasamento teórico para a operacionalização da vigilância do território de abrangência das unidades de saúde selecionadas, uma vez que a análise situacional não contempla só doenças, agravos e mortes, mas também as necessidades de saúde da população (Castellanos, 1997).

Em seguida, os estudantes vivenciam a realidade das ações de vigilância em saúde no município, desde investigações e inquéritos epidemiológicos até o processamento de dados. Porém, é fundamental que esse momento não seja estritamente técnico, mas que seja acompanhado de uma análise crítica das ações de vigilância do município, com base no referencial teórico já estudado.

O quarto momento pedagógico é a elaboração de um projeto de intervenção local mediante as análises realizadas nos momentos anteriores - voltado para diferentes atores do processo de vigilância compreendidos desde a atenção básica até o setor de vigilância em saúde - ou um projeto de intervenção voltado para a população de forma a empoderá-la das informações de saúde e dos principais agravos e riscos a sua saúde.

O quinto e último momento consta da operacionalização do projeto de intervenção que visa à transformação da realidade local. Uma vez que se reorienta o processo de trabalho dos atores envolvidos, ocorre melhora na qualidade das ações de vigilância em saúde; com isso, obtêm-se indicadores de saúde mais confiáveis que realmente expressem a realidade das condições 
de saúde da população, capazes de subsidiar o planejamento em saúde e o desenvolvimento de políticas públicas de saúde. Esse momento é, também, quando se dá o empoderamento da população sobre os determinantes e condicionantes socioambientais de sua saúde, com o controle de alguns fatores por parte dela, modificando, por sua vez, os indicadores de saúde.

Essa nova tecnologia permite o desenvolvimento das ações de vigilância integradas no território, entendendo que os problemas de saúde se apresentam de forma integral e que os fatores condicionantes deles não estão dissociados, e que a atuação em vigilância em saúde deve pautar-se num conhecimento abrangente com perspectiva do desencadeamento de ações interdisciplinares e intersetoriais, inclusive em um foco de promoção à saúde. Para Mendes (1996), quando a vigilância à saúde é focada no território, ela representa a possibilidade de se organizar o trabalho da equipe de saúde da família para enfrentar os problemas em diferentes períodos do processo saúde-doença.

Figura 1

Círculo tecnológico de educação em serviço na Vigilância em Saúde

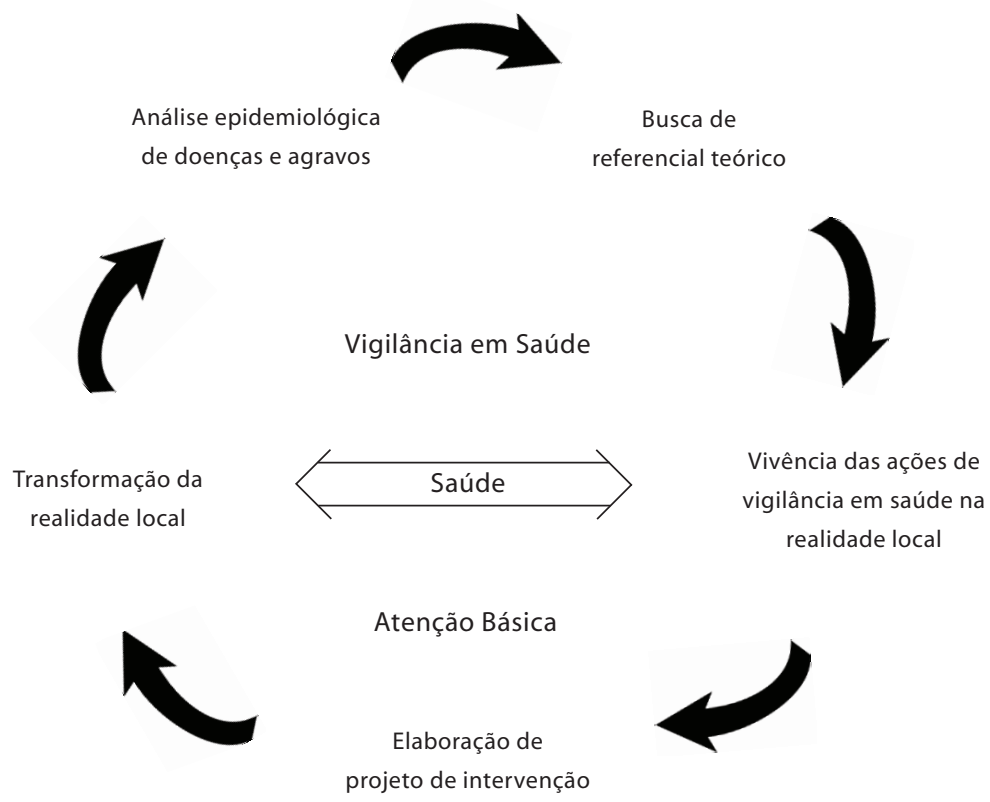

Fonte: Os autores. 


\section{Metodologia de avaliação e acompanhamento dos estudantes nas vivências em VS}

No processo de trabalho do PET-Saúde/VS de Sobral, elaborou-se um sistema de avaliação e acompanhamento dos estudantes. Cada bolsista constrói um diário de campo, que compreende um instrumento de compilação das vivências realizadas por ele durante o mês, reunindo registros de visitas, resumos, fichamentos, projetos, relatórios de pesquisa e, principalmente, ensaios autorreflexivos que permitam aos alunos a reflexão sobre sua prática.

Criou-se ainda um instrumento de avaliação da participação de cada bolsista, no qual era atribuída pontuação aos seguintes critérios: 'motivação' (direcionamento de capacidades e interesses do indivíduo, de modo dinâmico e constante, para o alcance dos resultados esperados, ou busca de novas responsabilidades); 'controle emocional' (manter domínio sobre relações emocionais diante de situações adversas); 'inteligência prática' (compreender e adaptar-se facilmente); 'adaptação' (integrar-se ao meio, com disposição positiva a mudanças, diferentes situações e novos contextos); 'assertividade' (responder adequadamente às situações sociais sem se sentir culpado); 'iniciativa' (iniciar uma ação por si mesmo, visando atingir objetivos previamente determinados); 'comportamento ético/sociabilidade' (capacidade de se relacionar bem com profissionais do serviço e a pessoa cuidada); 'assiduidade/pontualidade' (assiduidade e prontidão na realização das atividades propostas) e 'realização de atividades de pesquisa'. Além destes atributos, os estudantes foram avaliados quanto às competências relacionadas com as 'práticas de vigilância em saúde', como capacidade de obter as informações em vigilância utilizando recursos disponíveis e relacioná-las com a realidade, assim como de apontar estratégias para desenvolver ações que possibilitem minimizar, controlar, eliminar ou prevenir riscos à saúde.

O instrumento de avaliação permitiu analisar a capacidade de aprendizado dos estudantes em VS, bem como a sua competência para resolver problemas e instrumentalizar o conhecimento para a tomada de decisões.

\section{A experiência}

Inicialmente, durante os dois primeiros meses de vigência do projeto, optou-se por realizar uma imersão dos integrantes no serviço de vigilância em saúde local, com o objetivo de conhecer o processo de trabalho dos profissionais que ali atuam e se apropriar dele, além de reconhecer a importância da vigilância no processo saúde-doença de uma população. Momentos formativos e vivenciais foram propiciados para todos os integrantes, para que os 
diferentes saberes, da academia e do serviço, bem como dos diferentes cursos de graduação (medicina, enfermagem e odontologia), fossem ressignificados e potencializados para um campo comum, objetivando assim a interdisciplinaridade do conhecimento e a integração ensino-serviço, pois entende-se que a interdisciplinaridade é uma exigência para a integralidade (Minayo, 2000).

Desenvolveu-se uma formação teórica de forma dialógica sobre o que é vigilância em saúde, sobre os indicadores do Pacto pela Vida consensuados em Sobral - o qual representa os compromissos assumidos entre os gestores do SUS em torno de prioridades que impactam as condições de saúde da população -, e sobre os principais sistemas de informação, entendendo-os como ferramenta para a vigilância em saúde. Realizou-se também o estudo de manuais básicos sobre os indicadores de saúde.

Foram estudados os sistemas de informação gerenciados pela Coordenação de Vigilância à Saúde, que incluem: Sistema de Informação de Agravos de Notificação (Sinan), Sistema de Informações sobre Mortalidade (SIM), Sistema de Informações sobre Nascidos Vivos (Sinasc), Sistema de Acompanhamento do Programa de Humanização no Pré-Natal e Nascimento (Sisprenatal), Sistema de Cadastramento e Acompanhamento de Hipertensos e Diabéticos (Hiperdia), Sistema de Vigilância Alimentar e Nutricional (Sisvan), Sistema de Informação da Atenção Básica (Siab) e Programa de Monitorização das Doenças Diarreicas Agudas (MDDA) e Programa de Monitorização de Infecções Respiratórias Agudas (Mira). Este último programa foi elaborado e implantado pela Vigilância em Saúde do município de Sobral.

Os serviços da atenção básica de Sobral, como os Centros de Saúde da Família, por exemplo, são as principais fontes de dados sobre doenças e eventos relacionados à saúde para alimentar os sistemas de informação.

Outro momento formativo voltou-se para a realização de pesquisa científica, propiciando aos integrantes a formação para elaborar e executar projetos de pesquisa, bem como artigos científicos e resumos para apreciação em encontros científicos.

As vivências no serviço permitiram o exercício da construção dos principais indicadores de saúde. O primeiro produto trabalhado pelos estudantes nas vivências no serviço e nas pesquisas foi a análise da situação da sífilis em Sobral e da hanseníase, da Síndrome da Imunodeficiência Adquirida (Aids) e da tuberculose em seis territórios da atenção básica com maior relevância epidemiológica no município. Foram utilizados dados demográficos, socioeconômicos, de mortalidade, de morbidade, fatores de risco e de cobertura de cada área, referentes ao período de 2004 a 2009. Realizaram-se ainda discussões sobre os dados encontrados com o objetivo de exercitar o raciocínio epidemiológico e elaborar possíveis hipóteses explicativas para as situações de saúde encontradas nos territórios de atuação dos integrantes do PET-Saúde/VS. 
A inserção dos alunos no serviço de vigilância em saúde proporcionou conhecimento prévio sobre o funcionamento das áreas que este serviço abrange e como se articulam com todo o sistema de saúde local. Além disso, o conhecimento da possibilidade que os sistemas de informação têm de fornecer dados para subsidiar o trabalho da VS foi essencial para a iniciação das atividades previstas do projeto.

Os indicadores servem de suporte ao planejamento, administração e avaliação das ações de saúde. O conhecimento sobre a situação é essencial para estabelecer prioridades, alocar e gerir os recursos de forma positiva, melhorando as condições de saúde da população (Feuerwerker, 2000; Rouquayrol e Goldbaum, 2003).

Para se colocar em prática um modelo de assistência com base na VS, requer-se um profissional formado com base clínica, epidemiológica e humanística, pois só assim se pode esperar que esteja capacitado para agir diante das mais diversas situações (Mota e Carvalho, 2003; Bertolozzi e Fracolli, 2004).

Deve-se investir na organização de processos ensino-aprendizagem que propiciem ao aluno de saúde, mais do que se capacitar tecnicamente, que se torne agente ativo das mudanças que a sociedade brasileira necessita para que o país se torne mais equânime e justo. Outra potencialidade desse processo de ensino, articulado à realidade do sistema de saúde, é levar o estudante a construir (e não apenas consumir) o conhecimento mediante a articulação, in loco, entre teoria e prática, com base na experiência da interdisciplinaridade.

\section{Considerações finais}

No processo de implantação do programa, foi possível perceber o interesse e a facilidade dos estudantes na apropriação sobre o funcionamento dos sistemas de informações em saúde e sua utilização como fonte de recursos para a obtenção de informações em saúde para a tomada de decisão. Eles identificaram as fontes e os instrumentos de coletas para os bancos de dados dos sistemas de informações, bem como fluxogramas e protocolos utilizados na vigilância em saúde. É notória a compreensão dos estudantes sobre os conceitos utilizados na prática diária da epidemiologia e o reconhecimento da importância do monitoramento e da avaliação de indicadores pelos serviços de vigilância em saúde de forma integrada com a atenção básica.

A perspectiva do PET-Saúde/VS é a de promover a ampliação do entendimento das ações de vigilância em saúde nos serviços da atenção básica do SUS. Isso poderá implicar mudanças na forma de pensar e agir dos trabalhadores da saúde, uma vez que a organização das práticas ocorre na 'ponta' do sistema de saúde, isto é, no espaço onde eles interagem com a população e procuram atender às suas necessidades de saúde de forma integral. 
Essa experiência, além de ter proporcionado um conhecimento abrangente de fatores determinantes e condicionantes do processo saúde-doença/cuidado da comunidade local, mostrou aos estudantes, preceptores e tutores do Pet-Saúde/VS a importância da prática da vigilância em saúde para o diagnóstico de saúde da população. Evidenciou também que o processo de trabalho da vigilância, quando destacadas as peculiaridades em territórios específicos, conduz um planejamento racional e efetivo num território determinado, especialmente em microáreas da Estratégia Saúde da Família.

Com o desenvolvimento desse projeto, espera-se estimular o raciocínio e a sensibilidade dos alunos que participam do PET-Saúde/VS, de modo a utilizarem o conjunto de ferramentas e ações disponibilizadas pela vigilância em saúde em suas rotinas, principalmente de atenção à saúde coletiva (Pinto, 2008).

\section{Notas}

1 Universidade Estadual Vale do Acaraú, Sobral, Ceará, Brasil. Mestre em Enfermagem pela Universidade Federal do Ceará. <socorrocarneirol@gmail.com> Correspondência: Rua Desembargador Moreira da Rocha, 352, CEP 62010-140, Sobral, Ceará, Brasil.

2 Universidade Estadual Vale do Acaraú, Sobral, Ceará, Brasil.

Doutora em Enfermagem na Promoção da Saúde pela Universidade Estadual Vale do Acaraú. Coordenadora adjunta do Propet-Saúde/UVA. <cibellyaliny@gmail.com>

3 Universidade Federal do Ceará, Sobral, Ceará, Brasil.

Doutoranda em Saúde Coletiva pela Universidade Federal do Rio Grande do Norte. <anakarinemt@hotmail.com>

4 Prefeitura Municipal de Sobral, Sobral, Ceará, Brasil.

Graduado em Medicina Veterinária pela Universidade Estadual do Ceará.

$<$ netovet@sobral.org >

5 Prefeitura Municipal de Sobral, Sobral, Ceará, Brasil.

Graduada em Enfermagem pela Universidade Estadual Vale do Acaraú. Preceptora do PET-Saúde/VS. <sandra_flor38@hotmail.com>

6 Prefeitura Municipal de Sobral, Sobral, Ceará, Brasil.

Mestrando em Saúde da Família pela Rede Nordeste em Saúde da Família. Coordenador das ações de controle da tuberculose e hanseníase. Preceptor do PET-Saúde/VS. $<$ j.s32@hotmail.com> 
7 Prefeitura Municipal de Sobral, Sobral, Ceará, Brasil.

Mestre em Zootecnica pela Universidade Estadual Vale do Acaraú.

$<$ fcoroger@hotmail.com>

\section{Referências}

BERTOLOZZI, Maria R.; FRACOLLI, Lislaine A. Vigilância à saúde: alerta continuado em saúde coletiva. O Mundo Saúde, São Paulo, v. 28, n. 1, p. 14-20, jan./mar. 2004.

BRASIL. Ministério da Saúde. Gestão municipal de saúde: leis, normas e portarias atuais. Rio de Janeiro: Ministério da Saúde, 2001.

Ministério da Saúde. Comissão Nacional de Ética em Pesquisa. Resolução n. 196/96. Normas regulamentadoras de pesquisa envolvendo seres humanos. Brasília: Ministério da Saúde, CNS, 1996.

CASTELlAnOS, Pedro L. Epidemiologia, saúde pública, situação de saúde e condições de vida: considerações conceituais. In: BARATA, Rita B. (Org.). Condições de vida e situações de saúde. Rio de Janeiro: Abrasco, 1997. p. 31-75.

COSTA, Roberta K. S.; MIRANDA, Francisco A. N. Formação profissional no SUS: oportunidades de mudanças na perspectiva da Estratégia de Saúde da Família. Trabalho, Educação e Saúde, Rio de Janeiro, v. 6, n. 3, p. 503-517, nov.2008/fev.2009.

DIAS, Maria S. A. et al. Secretaria de Saúde de Sobral: construindo um sistema aprendente. Revista Sustentação, Fortaleza, n. 19, p. 32-33, abr./ jul. 2007.

FEUERWERKER, Laura C. M. A construção de sujeitos no processo de mudança da formação dos profissionais da saúde. Divulgação em Saúde para Debate, Londrina, n. 22, p. 18-24, dez. 2000.

MENDES, Eugênio V. Uma agenda para a saúde. São Paulo: Hucitec, 1996.

MERHY, Emerson E. Saúde: a cartografia do trabalho vivo. São Paulo: Hucitec, 2002.

MINAYO, Maria C. S. O desafio do conhecimento: pesquisa qualitativa em saúde. 7. ed. São Paulo: Abrasco; 2000.

MOTA, Eduardo L. A.; CARVALHO, Déa M. T. Sistemas de informação em saúde. In: ROUQUAYROL, Maria Zélia; ALMEIDA FILHO, Naomar de. Epidemiologia e saúde. 6. ed. Rio de Janeiro: Medsi, 2003. p. 605-628.

PINTO, Vicente P. T. et al. Análise do Processo de educação permanente para profissionais do SUS: a experiência de Sobral-CE. Sanare: Revista de Políticas Públicas, Sobral, v. 7, n. 2, p. 62-70, 2008.

ROUQUAYROL, Maria Zélia; GOLDBAUM, Moises. Epidemiologia, história natural e prevenção de doenças. In: ROUQUAYROL, Maria Zélia. Epidemiologia e saúde. 6. ed. Rio de Janeiro: Medsi, 2003. p. 17-35.

SETA, Marismary H., REIS, Lenice G. C. As vigilâncias do campo da saúde, o risco como conceito fundamental e a caracterização dos seus processos de trabalho. In: OLIVEIRA, Roberta G. (Org.) Qualificação de gestores do SUS. Rio de Janeiro: EAD/Ensp, 2009. p. 219-262. 
WALDMAN, Eliseu A. A vigilância como instrumento de saúde pública. In:

Vigilância em saúde pública. v. 7. Colaboração de Tereza Etsuko da C. Rosa. São Paulo: Faculdade de Saúde Pública da Universidade de São Paulo, 1998. (Série Saúde \& Cidadania). p. 91-132.

Recebido em 06/04/2011

Aprovado em 18/11/2012 